Revue Française de Civilisation Britannique

\title{
Les Think tanks néo-libéraux et les élections législatives britanniques de 2019
}

Neoliberal Think Tanks and the 2019 British General Election

Mark Garnett et Virgile Lorenzoni

\section{OpenEdition}

Journals

Édition électronique

URL : http://journals.openedition.org/rfcb/6027

DOI : $10.4000 /$ rfcb.6027

ISSN : 2429-4373

Éditeur

CRECIB - Centre de recherche et d'études en civilisation britannique

Référence électronique

Mark Garnett et Virgile Lorenzoni, « Les Think tanks néo-libéraux et les élections législatives

britanniques de $2019 »$, Revue Française de Civilisation Britannique [En ligne], XXV-3 | 2020, mis en ligne le 10 septembre 2020, consulté le 10 septembre 2020. URL : http://journals.openedition.org/rfcb/ 6027 ; DOl : https://doi.org/10.4000/rfcb.6027

Ce document a été généré automatiquement le 10 septembre 2020.

\section{(i) $\odot$

Revue française de civilisation britannique est mis à disposition selon les termes de la licence Creative Commons Attribution - Pas d'Utilisation Commerciale - Pas de Modification 4.0 International. 


\title{
Les Think tanks néo-libéraux et les élections législatives britanniques de 2019
}

\author{
Neoliberal Think Tanks and the 2019 British General Election
}

Mark Garnett et Virgile Lorenzoni

\section{Introduction}

1 Les think tanks situés à la droite du spectre politique britannique ont évolué au cours du temps, et leurs méthodes ont changé en conséquence. Originellement conçus comme des outils destinés à revitaliser la pensée conservatrice ou à former les élites du Parti conservateur, et inspirés de modèles traditionnellement associés à la gauche ${ }^{1}$, comme la Fabian Society, ils prennent leur forme actuelle dans les années cinquante avec la création de l'Institute of Economic Affairs (IEA). Dédié à la promotion de l'économie de marché, celui-ci voit le jour en 1955 sous l'impulsion de membres du Parti conservateur et du Parti libéral, mus par le constat partagé de la progression, perçue comme menaçante et quasi-hégémonique, d'une forme de pensée étatiste au Royaume-Uni. Puisant dans les travaux de Friedrich Hayek, Milton Friedman et Karl Popper, l'IEA contribuera à la formation intellectuelle de Margaret Thatcher et de sa garde rapprochée, et jouera un rôle certain dans la conversion du conservatisme britannique au libéralisme économique ${ }^{2}$.

2 Toutefois, à partir des années soixante-dix, ce modèle originel, reposant encore dans une certaine mesure sur des analyses économiques approfondies, sera graduellement effacé par l'apparition d'organisations fortement orientées idéologiquement, que nous qualifions ici de think tanks évidés ${ }^{3}$; des organisations plus intéressées par une couverture médiatique positive que par la production d'une recherche rigoureuse. Le modèle du think tank évidé repose sur un système agressif de promotion, une maitrise des tropes et des codes médiatiques, ainsi que sur la construction d'un discours simplifié, séduisant et trompeur, bâti autour d'une promesse d'innovation. En outre, et 
notamment sous l'impulsion d'Antony Fisher, le créateur de l'IEA, on observera à partir des années quatre-vingt une multiplication de ces institutions, toutes dédiées à la promotion d'idées néo-libérales, et fonctionnant en réseau 4 .

3 Nous examinerons ici les contributions faites par ces think tanks à la campagne électorale de 2019 au Royaume-Uni. Bien entendu, ceux-ci ne sont pas actifs qu'en période électorale, et, comme évoqué précédemment, ils travaillent depuis longtemps à la promotion de l'économie de marché et de la privatisation; ils ont notamment été particulièrement actifs dans le débat autour du Brexit ${ }^{5}$. Tout en gardant à l'esprit l'impact qu'ont eu ces think tanks sur le long terme, nous entendons circonscrire notre analyse au débat entourant l'élection législative de 2019, afin de proposer une évaluation de la pertinence de leur discours après la crise du Brexit, au moment où des institutions plus traditionnelles, comme l'Institute of Fiscal Studies (IFS), produisent des analyses plus équilibrées, qui ont été traitées avec un respect considérable dans les médias et dans les récents débats économiques. Nous proposons donc ici une étude des contributions au débat électoral de l'IEA (qui semble lui-même céder au modèle évidé) et d'autres think tanks libéraux plus récents, entre le 29 octobre 2019 (jour de l'approbation par le Parlement d'une élection législative anticipée) et le 12 décembre, date du scrutin.

4 Nous avançons l'idée que les think tanks évidés ne sont pas aussi adaptés à l'ère du Brexit et de la politique populiste que l'on pourrait le supposer. En effet, les think tanks idéologiques ont-ils toujours un rôle utile à jouer, au sein d'un discours de plus en plus polarisé entre la présentation des faits et la diffusion d'informations fortement orientées? Le principal problème rencontré par ces organisations avant l'élection était le fait que le manifeste du Parti conservateur, bien que dominé par le thème du Brexit et le concept de Global Britain, contenait des propositions d'aide publique et de nationalisation; des mesures auxquelles ces think tanks ont coutume de s'opposer fortement. On peut alors se demander si leur orientation idéologique réduit leur capacité d'adaptation, les rendant incapables de suivre la trajectoire plus stratégique empruntée par le Parti conservateur de Boris Johnson.

\section{Le cas de l'Institute of Economic Affairs}

La première étape consiste logiquement à s'intéresser au discours de l'IEA à l'approche des élections de 2019, car il représente, comme nous l'avons vu, le prototype du think tank néo-libéral britannique, tout en tentant de conserver la rigueur académique et l'indépendance qui ont fait sa réputation. Dans une élection où l'enjeu principal était le Brexit, il semble naturel de s'intéresser aux contributions de l'IEA sur ce sujet précis. Un an après le référendum de 2016, l'IEA établit une Brexit Unit, dirigée par Julian Jessop, dont la tâche est de publier divers «briefings » sur de multiples aspects du sujet, avec l'objectif principal de promouvoir le libre-échange en décrivant le Brexit comme «a once-in-a-generation opportunity to create a more flexible, open and vibrant economy and set a shining example for other countries ${ }^{6}$ ". L'idée même de "briefings" suggère un format compact, sciemment conçu pour exercer une influence immédiate dans le débat politique. Cependant, la Brexit Unit n'enregistre aucune publication entre l'annonce de l'élection anticipée et l'élection elle-même. Cela s'explique par la nécessité quasi-vitale pour les think tanks de demeurer officiellement éloignés de toute recherche partisane; en effet, beaucoup de ces institutions bénéficient d'un statut 
particulier, octroyé par la Charity Commission, qui permet à leurs donateurs de bénéficier de réductions d'impôts. Ce statut est donc synonyme de stabilité financière et d'attractivité pour les donateurs. Il arrive parfois que celui-ci soit menacé : l'IEA a ainsi récemment reçu plusieurs avertissements légaux quant à leur activisme politique dans le débat sur le Brexit ${ }^{7}$. Dans une élection où le slogan du Parti conservateur était " Get Brexit Done ", poursuivre une stratégie agressive en faveur du Brexit peut évidemment être interprété, à raison, comme du militantisme en faveur du parti de Johnson, et représenter un risque pour la stabilité financière des think tanks.

6 L'IEA est cependant loin d'avoir été inactif durant la campagne. L'un des indicateurs de performance favoris des think tanks cherchant à auto-évaluer leur influence consiste à compter leur nombre citations dans les médias. La pertinence de cette méthode reste sujette à caution, puisqu'elle donne l'impression que toute publicité, bonne ou mauvaise, peut être considérée comme une contribution utile au débat public. On peut illustrer les limites d'une telle méthode en faisant observer que certaines de ces citations, dans le cas de l'IEA, proviennent par exemple du New Statesman, qui les utilise pour illustrer certaines incohérences entre leurs propositions libérales et le manifeste du Parti conservateur8.

7 Toutefois, les médias ont joué un rôle crucial dans la campagne, et la présence constante de la voix des think tanks néo-libéraux dans les médias conservateurs peut être analysée comme un indicateur d'une influence possible sur l'opinion publique. Certains observateurs avancent l'argument que la même idée, répétée en boucle par des sources multiples (commentateurs et experts, qui, dans le cas qui nous intéresse, sont membres de think tanks), agissant sous des bannières différentes mais entretenant en réalité des liens étroits (nombre de think tanks néo-libéraux font partie d'une superstructure, l'Atlas Network, créé par Antony Fisher au début des années quatrevingt, sur lequel nous revenons plus bas) peut créer l'illusion d'un discours dominant, qui trouve artificiellement son chemin au cœur de la politique conventionnelle à coups de matraquage médiatique. Andrew Simms, co-directeur de la New Weather Foundation, un think tank dédié aux questions environnementales, décrivait déjà ce phénomène quelques mois avant l'élection :

[b]y encouraging the creation of more and more thinktanks - a never-ending production line of new "institutes", "centres" and "foundations", whose acronyms blur into each other - the network can generate a "constant river of commentary" [...]. A predominantly right-wing British media have been happy to give them space. This gives the impression of widespread support for what may be minority or fringe points of view9.

8 Ainsi, l'influence des think tanks néo-libéraux, bien que difficile à quantifier, bénéficie sans aucun doute de l'emploi de ce type de stratégie.

9 Il faut noter que les membres de l'IEA ont été particulièrement actifs dans les médias durant la campagne. On dénombre presque une citation par jour, même si certaines proviennent des mêmes interviews, articles, ou rapports. Deux grands thèmes émergent parmi les préoccupations des experts de l'IEA : tout d'abord, le Brexit, bien qu'abordé de manière détournée, via la double perspective du libre-échange et de l'intervention $\mathrm{du}$ gouvernement dans l'économie (un nombre important d'interventions sont consacrées à des questions de nationalisation ou de renationalisation, au NHS et à sa possible inclusion dans un accord de libre-échange entre le Royaume-Uni et les Etats-Unis, et même à l'aide extérieure).En parallèle, de nombreuses critiques sont formulées à l'égard des programmes de dépenses du Parti 
travailliste et du Parti conservateur, qui manquent, selon l'IEA, d'un sens de la « responsabilité fiscale (fiscal responsibility)».

\section{Reformuler la question du Brexit : Global Britain et libre-échange}

10 Il paraît évident que le Brexit ne pouvait être absent du débat électoral de décembre 2019, et l'IEA, malgré les difficultés évoquées plus haut, ne pouvait passer à côté d'un tel sujet. Si l'on considère la position des think tanks néo-libéraux, très largement favorables à la sortie du Royaume-Uni de l'Union Européenne, il est logique que beaucoup d'interventions de leurs membres évoquent de façon positive un hypothétique Royaume-Uni post-Brexit. Toutefois, deux angles sont privilégiés, afin de reformuler les termes du débat. Le premier est celui du libre-échange, qui déploie un discours présentant le Brexit comme une opportunité unique pour le Royaume-Uni de se libérer des chaînes de l'Union Européenne pour se transformer en Global Britain. L'idée est de propulser la nation "Out of Europe and into the world", si l'on emprunte le slogan de la campagne pour le « Non » du référendum sur la Communauté Économique Européenne de 1975.

11 Ce concept de Global Britain repose sur la réactivation possible de relations commerciales avec les pays de l'ancien Commonwealth, qui viendraient en théorie équilibrer, ou même dépasser, les pertes commerciales dues à la sortie de l'Union Européenne et du Marché Unique. Un aspect important de ce discours est l'insistance sur la compatibilité naturelle, presque organique, du Royaume-Uni avec ses anciennes colonies anglophones. L'IEA reprend en partie ces arguments : le 24 octobre, un peu en amont du débat électoral, Kate Andrews (directrice associée de l'IEA) faisait partie d'un panel d'experts pour $B B C$ Question Time; dans cette émission, elle insistait sur l'importance des relations commerciales hors Union Européenne : " 54 per cent of [the UK's] trade is done [outside the EU], trade is not a zero-sum game ${ }^{10}$. "

12 C'est dans ce contexte que nombre d'experts appartenant à l'IEA ont apporté leur soutien à un accord de libre-échange entre le Royaume-Uni et les États-Unis, présentés comme partenaire commercial le plus naturel, en défendant une redynamisation économique de la relation spéciale. Le $1^{\mathrm{er}}$ novembre, Shanker Singham, alors à la tête de la International Trade and Competition Unit de l'IEA, présente ce projet dans le Daily Telegraph. Dans son article, il défend l'accord de sortie obtenu par Boris Johnson en dépit des inquiétudes émises par Donald Trump, en avançant l'argument que cet accord représente une avancée significative par rapport à celui proposé par Theresa May, notamment en ce qui concerne l'ouverture de nouvelles possibilités pour un meilleur accord de libre-échange avec les États-Unis. La plupart des interventions de l'IEA sur ce sujet, toutefois, sont bien plus spécifiques : elles s'emploient à nier l'inclusion du NHS dans le cadre de ce possible accord de libre-échange, et à réfuter l'idée, pour reprendre les mots de Jeremy Corbyn, que le NHS est "up for sale ", après la diffusion de documents faisant état de pourparlers secrets entre les équipes de négociateurs de Downing Street et de la Maison Blanche.

13 Le 27 novembre, Jeremy Corbyn brandit ces documents devant les caméras. Immédiatement, l'IEA réagit dans différents médias. Kate Andrews, écrivant dans City A.M., nie toute preuve que le NHS est réellement « on the table » dans les négociations anglo-américaines. Elle écrit: "the hysteria being whipped up over the NHS is rooted in political messaging, not evidence ${ }^{11} »$, tirant ainsi la conclusion que rien, dans les 
documents produits par le leader travailliste, ne prouve que le NHS coure un réel danger. Le même jour, Victoria Hewson (directrice du pôle des affaires réglementaires de l'IEA), écrivant pour BrexitCentral (un blog créé par des membres de Vote Leave), fait une déclaration très similaire, tout en célébrant au passage un possible accord entre Londres et Washington : "[the documents] offer a fascinating insight into the discussions that [...] show a breadth of ambition and considerable commitment to the realisation of a Free Trade Agreement between the UK and the US ${ }^{12}$. "

L'argument final avancé par l'IEA sur ce sujet provient de Mark Littlewood, le Directeur Général de l'IEA. Dans sa colonne bimensuelle dans le Times, il écrit qu'un tel débat est stérile: la seule chose qui compte, de son point de vue, c'est de saisir toutes les opportunités pour améliorer le système de santé du pays. Pour Littlewood, «what the leaked documents do show is that the Americans are interested in removing barriers to facilitate more buying and selling across the Atlantic. This should be seen as an opportunity, not a threat ${ }^{13}$ ». Dans cet article, on retrouve l'argument central des think tanks néo-libéraux, à savoir l'introduction de la concurrence provenant du secteur privé comme panacée économique. En outre, Littlewood met à nouveau l'accent sur le Brexit comme opportunité unique pour le libre-échange.

\section{Brexit et nationalisation}

Le NHS, ainsi que la question du Brexit, ne sont pas abordés par l'IEA uniquement du point du vue global du libre-échange: les questions de nationalisation, souvent désignées par le vocable péjoratif de state grabs, sont également au centre de son discours. La nationalisation des infrastructures d'accès à l'internet haut débit proposée par le Parti travailliste va, par exemple, déclencher l'ire du think tank. Le 14 novembre, au lendemain de la révélation du projet, Littlewood est cité dans un article de City A.M. dévoilant la réaction de la City à cette nouvelle : " to bring internet provision into the state's remit would ensure all of the delays, waiting times and quality decline that go hand-inhand with bureaucracy ${ }^{14}$. » La même citation apparaît dans le Daily Mail, le même jour ${ }^{15}$. Cela révèle une autre stratégie des think tanks : ils utilisent une gamme variée de canaux d'informations, qui leur permet d'atteindre différentes audiences : le public très spécialisé de la City d'un côté, et le public britannique (voire parfois international) au sens large, de l'autre. La nationalisation d'Openreach, le réseau de British Telecom, est également évoquée par Julian Jessop dans le Daily Telegraph du 16 novembre, où il déclare que le financement du projet via des emprunts est fiscalement irresponsable : « [b]orrowing is still borrowing, whatever the purpose, and someone would still have to be willing to hold the new bonds ${ }^{16}$."

Les plans de nationalisation du Parti travailliste sont à nouveau attaqués par Kate Andrews dans un article à destination d'une audience plus populaire, dans le Sunday Express du 17 novembre. Selon Andrews, les projets de Corbyn pourraient, à terme, menacer les retraités, et finir par être en fait financés en grande partie par le contribuable. Dans son article, elle s'en prend à la vision travailliste de l'entreprise comme " a self-interested and opaque [...] bogeyman »; pour elle, "wealth creation generated by business is what makes delivering high-quality public services possible ${ }^{17} »$. Ces arguments familiers seront réutilisés dans un rapport publié par l'IEA sur son site internet quelques jours avant l'élection, le 9 décembre. Le rapport, co-écrit par Julian Jessop et Len Shackleton, est intitulé Renationalisation: Back to the Future?, et se veut porteur d'un 
avertissement: "the potential costs of nationalisation are huge and most of the potential benefits could be achieved in other ways ${ }^{18}$. $\gg$ Le rapport bénéficie d'une visibilité dans les médias : il est cité la veille de sa publication dans le Daily Telegraph ${ }^{19}$ et le Daily Mail ${ }^{20}$, et le lendemain dans le Times ${ }^{21}$.

Cette promotion médiatique tend à démontrer que l'IEA peut compter sur des journaux conservateurs comme le Daily Telegraph, ainsi que sur des tabloïds dont la sensibilité politique est à droite, pour diffuser ses idées et leur donner un poids important dans le débat public, en particulier en période électorale. Le reconditionnement et la diffusion d'idées néo-libérales est donc devenu l'un des objectifs principaux de ce type de think tank, et dénote un glissement vers le lobbying, souvent au détriment de la qualité de la recherche produite. L'IEA, malgré son statut de précurseur, n'échappe pas à cette tendance.

\section{La responsabilité fiscale comme stratégie d'indépendance}

18 Il faut également rappeler que l'IEA, malgré une hostilité supérieure envers le Parti travailliste, a égratigné à plusieurs reprises le Parti conservateur durant la campagne, notamment sur des questions d'imposition et de dépenses publiques. Le 26 novembre, Mark Littlewood, écrivant dans le Times, déclare qu'il reste encore un long chemin à parcourir aux Tories pour pouvoir être considérés comme le parti du contribuable. Il exprime ses doutes quant au tax triple lock, inclus dans le manifeste conservateur, qui verrouille les taux de base de l'impôt sur le revenu, des contributions à la sécurité sociale, et de la TVA. Pour lui, certaines dérives inquiétantes sont possibles, par exemple si les taux en viennent à monter dans des domaines annexes, non couverts par les promesses conservatrices ${ }^{22}$.

Il n'est pas surprenant qu'un think tank comme l'IEA attaque le Parti travailliste et son manifeste, ce dernier contenant des engagements très prononcés en faveur de l'intervention de l'état (entre autres, on peut citer un plan pour stopper la privatisation du NHS, la mise en place d'une commission sur l'intégration des écoles privées dans le système national, et la nationalisation des chemins de fer, des infrastructures permettant l'accès à l'internet haut débit et des services postaux; tout cela pour un coût estimé de 82,9 milliards de livres par an ${ }^{23}$ ). Une dimension plus intéressante de la participation de l'IEA dans le débat électoral est probablement sa critique du manifeste du Parti conservateur, qui est également décrit comme proposant une approche dangereusement étatiste. Même si les plans des conservateurs en matière de dépenses publiques (une augmentation annuelle de 2,9 milliards de livres ${ }^{24}$ ) peuvent être considérés comme modestes par rapport à ceux des travaillistes, les experts de l'IEA s'inquiètent de ces choix, en particulier en ce qui concerne le NHS et l'approche de la fiscalité.

La question de la responsabilité fiscale constitue donc un autre sujet d'inquiétude de l'IEA durant la campagne. Naturellement, les plans gargantuesques du Parti travailliste constituent la cible de plus d'une attaque de la part des think tankers : Len Shackleton, Julian Jessop et Mark Littlewood expriment tous leur incrédulité face aux dépenses prévues par Jeremy Corbyn ${ }^{2526}$, allant jusqu'à affirmer qu'elles pourraient mettre en danger les services publics, et laisser les futures générations criblées de dettes. Mais la critique de l'IEA s'étend au Parti conservateur : un nombre significatif de publications incluent une remise en question de leur plan de dépenses. Kate Andrews ouvre le bal le 
4 novembre dans une interview pour le Daily Express: «[Conservatives and Labour are] splashing the cash in a bid to win votes ${ }^{27}$." Ainsi, dès le début de la campagne, les conservateurs sont dans la ligne de mire de l'IEA.

21 De nombreuses autres publications traitent de ce même "cash splashing ": Kate Andrews réitère son propos dans City A.M. le 8 novembre : " [Even if] Labour promises are staggering, [The Conservatives are also] taking part in their own rule-loosening activity, to make way for the party's promises on the NHS, police numbers, and education spending ${ }^{28}$. " Elle nuance toutefois son jugement, en notant que les conservateurs méritent, de son point de vue, une certaine reconnaissance pour avoir contrôlé les finances publiques à l'issue de la crise de 2008, bien que cela ne les autorise pas à se lancer dans une frénésie de dépenses. C'est dans un article publié sur 1828 (un site d'opinion à forte tendance néolibérale) le 9 décembre, que la critique des Tories par l'IEA se fait la plus explicite. Emily Carver (à la tête du pôle média du think tank) explique que les conservateurs ne doivent pas être tentés par le big government, et devraient plutôt se faire les champions du capitalisme et du libre-échange; recourir à tout étatisme équivaudrait à une trahison des principes qu'ils défendent traditionnellement ${ }^{29}$. Bien entendu, elle nuance également ses accusations, et dans l'ensemble, la plupart des évaluations du programme de Boris Johnson demeurent globalement positives. Toutefois, les libéraux de l'IEA émettent un avertissement très clair: si leurs principes venaient à être menacés par l'un ou l'autre des partis, ils seraient alors prêts à en découdre.

Ces observations mènent à deux conclusions : tout d'abord, l'IEA fait preuve d'une bien plus grande virulence envers le Parti travailliste, mais maintient son indépendance visà-vis des Tories en critiquant certains aspects de leur programme. Ensuite, ces deux postures montrent l'attachement de l'IEA aux principes du libre-échange. Cette approche relativement équilibrée aurait pu être menacée si le think tank s'était concentré sur la question du Brexit de façon directe, amenant l'IEA sur un terrain où il aurait été dangereusement aligné avec le Parti conservateur et son mantra, «Get Brexit Done ». Cela permet d'expliquer la réserve de la Brexit Unit durant la campagne, et la reformulation du débat en des termes différents.

\section{Le conservatisme One Nation, menace pour les think tanks néo-libéraux}

Alors que les programmes des deux principaux partis permettent à l'IEA d'affirmer son indépendance, la nature du débat économique de la campagne de 2019 se révèle être un sérieux obstacle à sa soif de mener la bataille des idées à travers le prisme du libreéchange. En effet, le Parti conservateur de Johnson, dans sa tentative de séduire les électeurs travaillistes désenchantés dans les régions des Midlands ou du Nord de l'Angleterre, adopte des propositions de politiques publiques que Johnson lui-même identifie comme appartenant à la tradition du conservatisme One Nation; comme dans le Sybil, or the Two Nations de Disraeli, le Parti conservateur souhaite prouver que l'on peut lui faire confiance pour défendre les intérêts de la classe ouvrière. Outre l'instrumentalisation des thèmes de l'identité et de la souveraineté nationales, cette approche intègre un certain nombre d'engagements en termes de dépenses publiques, dans le but d'empêcher la détérioration des conditions de vie des classes les plus modestes, et d'inverser le déclin des services publics, deux processus déjà bien engagés depuis la crise financière de 2008. 
24 Les stratégies adoptées par le Parti conservateur lors de la campagne de 2019 représentent donc un défi important pour les think tanks comme l'IEA. De toute évidence, l'IEA conserve une synergie bien plus grande avec le Parti conservateur qu'avec les travaillistes, mais leur principale source d'entente réside dans le Brexit et les opportunités qu'il pourrait offrir pour une Global Britain fondée sur le libre-échange : ce thème étant devenu le point central de la campagne, il était donc plus difficile pour eux de promouvoir une telle idée sans sembler travailler directement dans l'intérêt des conservateurs, et donc risquer de perdre leur statut d'organisation caritative. Depuis l'arrivée de Margaret Thatcher à la tête du parti en 1975, l'IEA pouvait en effet ressentir une affinité avec le parti Tory grâce à leur cause commune, le libéralisme économique, tout en conservant une certaine indépendance. En effet, bien qu'ayant participé à la conversion du parti à la cause du libre-échange, ils pouvaient continuer à le critiquer de l'extérieur pour ses tentatives trop limitées de libérer l'économie britannique de toute forme d'interférence étatique. Avec Boris Johnson aux manettes, le danger réside dans le fait que l'indépendance de l'IEA puisse soudain devenir une réalité bien plus tangible : le parti pourrait adopter des politiques économiques incompatibles avec les principes de l'IEA, politiques représentant non plus une simple manœuvre tactique, mais bien une importante reconfiguration de la pensée conservatrice, aliénant ainsi définitivement le think tank.

\section{L'élection de 2019 comme révélateur d'une relation spéciale de l'ombre?}

25 Avant de se tourner vers les contributions d'autres think tanks néo-libéraux au débat électoral, il est nécessaire de replacer ceux-ci dans le réseau plus large auquel ils appartiennent. Antony Fisher, fondateur de l'IEA, après avoir compris l'influence potentielle d'organisations comme la sienne, travaille à partir de la fin des années soixante-dix à établir un réseau de think tanks similaires dans le monde entier. Cela mènera à la création de l'Atlas Network en 1981, qui inclut dès le départ d'autres institutions imaginées par Fisher, comme le Manhattan Institute for Policy Research (d'abord connu sous le nom d'International Center for Policy Studies, créé en 1977) ou le Pacific Institute for Public Policy (créé en 1979) ${ }^{30}$. Cette superstructure composée de think tanks néo-libéraux se doit d'être mentionnée ici, car elle a probablement joué un rôle dans la formulation des termes du débat autour du Brexit, et probablement exercé une influence sur l'opinion publique à l'approche du référendum de 2016; un rôle qu'elle reprend en partie lors de l'élection de 2019.

En effet, la construction intellectuelle et la promotion d'une Global Britain, conditions sine qua non à la prospérité d'un Royaume-Uni post-Brexit selon les think tanks libéraux, sont copieusement alimentées par les activités de l'Atlas Network, qui s'étendent bien au-delà des frontières nationales. Par exemple, la perspective d'un accord de libre-échange transatlantique est longuement étudiée dans un rapport publié un an avant l'élection, sous l'impulsion du Cato Institute (un important think tank conservateur américain) et de l'Initiative for Free Trade (IFT, créé en 2017 par l'ancien député européen conservateur Daniel Hannan). Le rapport, intitulé The Ideal UK-US FreeTrade Agreement: A Free Trader's Perspective $e^{31}$, est signé par onze think tanks néo-libéraux, parmi lesquels on retrouve, du côté britannique, l'IEA, le Centre for Policy Studies (CPS), et l'Adam Smith Institute (ASI). Le rapport bénéficie de la collaboration de 
plusieurs think tanks conservateurs américains, dont la Heritage Foundation (l'un des plus puissants think tanks conservateurs américains) le Manhattan Institute, et l'American Enterprise Institute. Toutes ces organisations sont membres, ou plutôt « partners ", de l'Atlas Network ${ }^{32}$. Cela démontre comment les think tanks néo-libéraux peuvent fonctionner en réseau, presque à la manière d'un écosystème, et les résultats qu'une telle stratégie peut fournir.

Il est possible d'avoir un aperçu de la manière dont cet entrelacs d'institutions a pu avoir un impact sur les thèmes centraux de l'élection législative. Un mois avant le début de la campagne, l'IEA et Taxpayers' Alliance (TPA, un groupe de pression créé par Matthew Elliot, qui dirige Vote Leave à partir de 2015) organisent ce qu'ils appellent un "Think Tent ». Deux séminaires donnés à cette occasion sont particulièrement dignes d'intérêt: Sajid Javid, alors encore Chancelier de l'Échiquier, est invité à prendre la parole le $1^{\mathrm{er}}$ octobre $^{33}$; un peu plus tôt le même jour, une discussion est organisée qui réunit non seulement des membres de l'IEA et de TPA, mais également Ted Bromund, membre de la Heritage Foundation (et également l'un des auteurs de l'Ideal UK-US Free Trade Agreement). La discussion s'intitule «Trade after Brexit - Will freer markets enrich $u s^{34}$ ? ». Ces interactions entre think tanks permettent de révéler, dans une certaine mesure, comment les idées peuvent circuler au sein d'une même superstructure avant d'être diffusées dans le but d'influencer à la fois les décideurs politiques et l'opinion publique. On peut également parler avec prudence d'une relation spéciale de l'ombre, dont les contours apparaissent et se confondent avec ceux de l'Atlas Network.

\section{Le Centre For Policy Studies}

Comme démontré plus haut, l'IEA est loin d'être le seul think tank à tenter d'exercer une influence sur le débat politique, et donc sur la campagne électorale. Le CPS est une autre institution qu'il est nécessaire de mentionner, puisqu'il représente un type différent de think tank, bien plus proche du Parti conservateur, et ne bénéficiant donc pas d'une inscription à la Charity Commission. Connu pour avoir été l'instrument politique de Thatcher en raison de sa capacité à produire des propositions de politiques publiques immédiatement applicables par un cabinet conservateur en exercice, il possède donc une plus grande marge de manœuvre lorsqu'il s'agit de proposer des analyses plus clairement alignées sur la vision et le programme politique des Tories. Toutefois, sa contribution au débat électoral est restée relativement limitée, que ce soit du point de vue de la recherche ou de l'activité médiatique.

Le CPS publie un rapport le 29 octobre, le jour de l'annonce de l'élection. Cette simultanéité peut sans doute être attribuée aux hasards du calendrier, mais le rapport est néanmoins intéressant dans son contexte, puisqu'il entend explorer " the full extent to which wealth, talent, trade and investment are concentrated in London and the South East ${ }^{35}$ " et propose un plan détaillé pour répartir plus largement la prospérité économique. Cet effort pour réduire l'écart entre le Sud-Est et les régions du nord de l'Angleterre correspond à la volonté des conservateurs de séduire les électeurs de régions historiquement dominées par les travaillistes. Le rapport insiste sur un point: «our United Kingdom does not always feel as united as we might like.» Outre la dimension euphémistique d'une telle phrase dans le contexte du Brexit, on observe là encore la vision presque disraélienne de deux nations qu'il faudrait réconcilier. 
30 Le CPS produit d'autres rapports en lien direct avec la campagne : l'un deux, publié le 5 novembre, est une attaque directe contre la proposition travailliste de mettre en place une semaine de quatre jours, sans perte de revenus ${ }^{36}$. Jethro Elsden, l'auteur du rapport, est plus que sceptique : " [t]he estimated cost to the public sector alone is $£ 45$ billion on current productivity levels, falling to $£ 17$ billion assuming extremely generous productivity gains from shorter hours ${ }^{37}$. » En un mot, le CPS rejoint la bataille pour la responsabilité fiscale, en dénonçant, comme l'IEA, un « cash splashing » de la part du Parti travailliste, dans le simple but de gagner des voix. Toutefois, dans ce cas précis, la critique ne s'étend pas au Parti conservateur. Les interventions du CPS dans les médias, peu nombreuses, critiquent uniquement le Parti travailliste (le rapport en question est par exemple cité dans le Times le 5 novembre ${ }^{38}$ ), probablement en raison de l'inutilité tactique pour le CPS d'équilibrer son discours. En outre, la visibilité très limitée du CPS dans le débat électoral semble le contredire lorsqu'il se décrit comme "the most influential think tank among Conservative $\mathrm{MPs}^{39}$ ».

\section{«The man who brought you Brexit » et l'Initiative for Free Trade}

31 L'Initiative for Free Trade, contrairement à l'IEA et au CPS, est un groupe relativement récent, créé par Daniel Hannan en septembre 2017. Il promeut de façon radicale les principes traditionnels des think tanks néo-libéraux, avec une insistance toute particulière sur le Brexit : "IFT makes the intellectual and moral case for free trade, and sees Britain's withdrawal from the European Union as a unique opportunity to revitalise the world trading system $^{40}$. " l'IFT est donc l'un des principaux promoteurs du concept de Global Britain, avec une contribution importante sous la forme de l'Ideal UK-US Free Trade Agreement mentionné plus haut. Hannan reconnaît volontiers avoir un intérêt de longue date pour la question européenne. Sam Knight, dans un article publié dans le Guardian en 2016, le présente déjà comme "the man who brought you Brexit ${ }^{41}$ ", et insiste sur son travail en coulisses pour réaliser ce dont il rêvait déjà en tant qu'étudiant, alors qu'il assistait à la démission forcée de Margaret Thatcher, deux ans après son discours de Bruges, et vingt-trois jours avant que John Major ne signe une première version du Traité de Maastricht.

32 L'article de Knight souligne également le fait que Hannan, bien que peu visible au sein de la campagne du Leave, s'est en fait très tôt employé, notamment à travers un réseau de think tanks et de groupes de pression, à rendre possible sa vision du Brexit. Son influence personnelle remonte d'ailleurs très loin, puisque, comme l'écrit Knight : «[i]t was Hannan, in 2012, who asked Matthew Elliott, the founder of the Taxpayers' Alliance, to set up the embryonic campaign group that later became Vote Leave. " Là encore, on peut voir se dessiner les contours d'un réseau d'institutions interconnectées, et on peut avancer l'idée que c'est en partie l'action collective de ces organisations qui a contribué à la formation du climat politique menant à l'élection anticipée de 2019, et ainsi à la victoire écrasante du Parti conservateur. L'IFT peut donc être considéré comme l'un des derniers stades de développement du think tank néo-libéral, qui propose une vision très étroite du Brexit comme panacée via le libre-échange.

Les contributions de l'IFT au débat électoral confirment cette interprétation. La veille de l'annonce officielle de l'élection, Hannan publie un article consacré aux bienfaits du libre-échange dans le Washington Examiner, où il critique les politiques protectionnistes 
de Donald Trump; il y déclare que le Royaume-Uni est bien mieux disposé au libéralisme économique que la plupart des pays, tout en abritant ce qu'il appelle son " anti-trade constituency", qu'il rejette en bloc comme un seul groupe de pression regroupant les anti-capitalistes, les anti-américains, les anti-Trump, les anti-Brexiteers, et les activistes végans ${ }^{42}$. Cet article a lui seul donne une idée des extrémités atteintes par certains think tanks britanniques dans leur rhétorique en faveur d'un Brexit libéral, et révèle là encore les liens étroits que ceux-ci entretiennent avec leurs équivalents américains (Hannan, dans son article, appuie sa critique des politiques protectionnistes de Trump sur des analyses de la National Taxpayers Union Foundation, un autre membre de l'Atlas Network ${ }^{43}$ ).

L'IFT s'emploie également à démentir les allégations de Jeremy Corbyn concernant l'inclusion du NHS dans les négociations en vue d'un accord de libre-échange transatlantique, en relayant sur son site internet l'article de Victoria Hewson mentionné plus haut. L'essentiel de ses contributions à la campagne, sans grande surprise, concernent le libre-échange, et plus particulièrement cette possibilité d'accord transatlantique. Le 5 novembre, un article de Nile Gardiner, le directeur du Margaret Thatcher Centre for Freedom de la Heritage Foundation, est publié dans le Daily Telegraph et relayé sur le site de l'IFT. L'article fait office de véritable prophétie autoréalisatrice : pour Gardiner, il y aura sans l'ombre d'un doute un accord de libreéchange d'ici 2020, malgré les réticences de Trump quant à l'accord de sortie négocié par Boris Johnson. Gardiner écrit :

President Trump has long been a huge supporter of a trade agreement with the UK. Having met and spoken with numerous US Administration officials, I am in no doubt that the Government there is 100 per cent committed to moving forward swiftly with a UK trade deal, ideally in $2020^{44}$.

La campagne de l'IFT est donc entièrement dédiée à la promotion du libre-échange, notamment dans le cadre bien précis d'une relation commerciale redynamisée entre le Royaume-Uni et les États-Unis.

\section{Conclusion}

Que révèle le rôle joué dans l'élection de 2019 par trois think tanks comme l'IEA, le CPS et l'IFT quant à leur pertinence dans une ère post-Brexit ? Tout d'abord, on peut faire valoir que ces think tanks et leur action au sein d'une structure globale ont participé à instaurer le climat politique menant au Brexit, et donc à l'élection de 2019. L'enthousiasme avec lequel les groupes de pression situés à la droite du spectre politique ont œuvré à la promotion du Brexit, particulièrement depuis la perspective du libre-échange, peut expliquer comment des idées marginales ont graduellement trouvé leur chemin au cœur de la politique mainstream. Comme le dirait Madsen Pirie, membre fondateur de l'Adam Smith Institute, ces idées, grâce à des think tanks comme le sien, ont été propulsées "from the edge of lunacy to the edge of policy" ${ }^{45}$ ». Il ne faut cependant pas totalement céder à un récit que l'on pourrait qualifier de quasitéléologique, qui reviendrait à valider sans réserve aucune la thèse des think tanks, qui clament haut et fort leur irrésistible influence. Au contraire, la participation des think tanks néo-libéraux à la campagne de 2019 permet également de démontrer que leur nature évidée pourrait en définitive se révéler un désavantage dans le nouveau paradigme politique qu'ils ont eux-mêmes contribué à construire. 

aient fréquemment pris la parole ou fait l'objet de citations dans les médias, une très grande partie de ces interventions restent circonscrites aux médias traditionnellement associés à la droite. Sur des plateformes plus équilibrées en termes idéologiques, comme la BBC, les analyses les plus fréquemment relayées étaient celle de l'Institute for Fiscal Studies (IFS), un think tank dont l'identité peut être qualifiée de trans-partisane, notamment en raison de son histoire (créé en 1965 par des experts financiers, dont un futur membre du Parti conservateur, William Hopper, il comptera également parmi ses directeurs Roy Jenkins, alors leader adjoint du Parti travailliste). L'IFS est parvenu, grâce à la qualité technique de ses publications, à produire une critique de chaque programme politique, tout en restant à l'abri de toute accusation de biais idéologique ou d'intérêts partisans; leurs analyses apparaissant jusque dans le Times $^{46}$. Par contraste, c'est précisément en raison de leur fort parti-pris idéologique en faveur du libre-échange et de l'économie de marché que beaucoup des membres des think tanks libéraux comme l'IEA ont reçu une couverture médiatique.

Si les arguments économiques utilisés dans la campagne de 2019 ne font que souligner la difficulté pour les think tanks libéraux comme l'IEA de maintenir leur indépendance dans un débat extrêmement polarisé, il existe un autre paramètre que ces institutions ont probablement sous-estimé, et qui permet de se poser la question de leur pertinence post-Brexit. Ces organisations, qui se concentrent presque exclusivement sur le libreéchange et sur la façon dont le Brexit pourrait être utilisé comme une opportunité pour plus de dérégulation, sont bien moins à l'aise avec les questions identitaires qui soustendaient le résultat du référendum de 2016. Bien que l'on puisse déceler, dans le concept de Global Britain, certains ingrédients du nationalisme anglais, la célébration du libre-échange par les think tanks comme l'IEA se marrie très difficilement avec l'approche de la souveraineté et de l'état adoptée par les conservateurs. Plus précisément, la stratégie de Johnson lors de l'élection, en empruntant à la tradition paternaliste $d u$ conservatisme One Nation, bien que très efficace dans les circonscriptions ayant voté Leave en 2016, contredit en de nombreux points les principes centraux des think tanks libéraux. En 2019, les conservateurs ont été capables d'adapter leur discours pour envoyer un message populiste, en sous-entendant que le Brexit pouvait signifier un retour de la souveraineté nationale comme le moyen ultime de «reprendre le contrôle (Taking Back Control)». Les think tanks néo-libéraux, dont l'identité s'est progressivement cristallisée en une forme thatchérienne de conservatisme, risquent d'avoir de grandes difficultés à s'adapter si le Parti conservateur persiste dans cette voie.

Au moment où nous écrivons ces lignes, il est difficile d'être certain que la campagne électorale de 2019 constitue bien un tournant idéologique majeur au sein du Parti conservateur. Sommes-nous à la fin d'un cycle ? Le conservatisme britannique est-il en train de subir une véritable mutation, profitant de son indéniable adaptabilité en tant qu'idéologie? Dans ce cas, les think tanks néo-libéraux, prisonniers d'une structure idéologique bien plus rigide, se trouveraient à nouveau dans la position qui était la leur dans les années cinquante, lorsqu'ils étaient les champions solitaires et marginaux du libre-échange. Jusqu'à présent, ils parviennent à maintenir l'impression qu'ils exercent une influence considérable sur le processus d'élaboration des politiques publiques (et pas seulement grâce à leur proximité avec certains hommes politiques conservateurs de premier plan). Cette impression leur permet de retenir l'attention des médias, et les 
faveurs de leurs donateurs. Tout indice de perte progressive d'influence pourrait avoir un impact considérable sur leurs financements, ainsi que sur leur capacité à diffuser leur message au sein de l'opinion publique grâce à la presse populaire.

En mars 2020, en pleine crise du coronavirus, alors que de nombreuses nations à travers le monde semblaient redécouvrir l'importance d'un système de santé national, Boris Johnson déclarait dans une conférence de presse : «there really is such a thing as society ${ }^{47}$. " C'était une contradiction volontaire de la remarque bien connue faite par Margaret Thatcher lors d'une interview pour le magazine Women's Own en 1987: " there's no such thing as society ». Hasard ou non, c'est également en 1987 que les Tories gagnent pour la dernière fois une majorité comparable à celle obtenue par le parti de Boris Johnson en 2019, avec sa marge de 80 sièges. Si la remarque de Thatcher a souvent été utilisée hors contexte, elle reflétait néanmoins une vision atomiste de la société (à l'exception importante des familles), dans laquelle la non-intervention de l'état était la condition centrale à l'existence de la liberté.

42 Johnson n'est pas le premier homme politique conservateur à prendre ses distances avec cette phrase, mais il existe de bonnes raisons de penser que ses mots avaient un sens, et qu'il devra agir en conséquence dès la fin de la pandémie. Par exemple, la gestion de la crise par le cabinet suggère qu'il pourrait graduellement modifier son approche du Brexit, notamment pour s'assurer que les conditions de vie des classes les plus modestes ne soient pas trop violemment touchées. Si le gouvernement de Johnson se tourne vers le populisme et le protectionnisme, plutôt que de saisir l'opportunité pour le libre-échange ouverte par le vote de 2016, l'aliénation des think tanks libéraux sera totale. Ironiquement, une telle trahison pourrait même, sur le long terme, revitaliser des institutions comme l'IEA : celles-ci, après des années passées à mener la bataille des idées sur tous les fronts, auraient alors d'autant plus de raisons de mener une contre-offensive agressive, retrouvant ainsi la place marginale et la saveur d'originalité qui avaient participé, dans les années soixante-dix, au triomphe de leurs idées.

43 Mark Garnett est maître de conférences en Politique et Relations Internationales à l'Université de Lancaster, et auteur de nombreux articles et ouvrages sur la politique britannique. Ses domaines de recherche concernent principalement la relation entre les idées et la pratique politique, le Parti conservateur britannique, et les think tanks.

44 Virgile Lorenzoni est agrégé d'anglais et doctorant à Aix-Marseille Université, sous la direction d'Isabelle Vagnoux et de Valérie André (LERMA). Sa thèse s'intitule: "Les think tanks conservateurs: étude de la production, de la diffusion et de l'influence des idées en politique britannique et américaine ». 


\section{BIBLIOGRAPHIE}

BERTHEZÈNE Clarisse, Training Minds for the War of Ideas: Ashridge College, the Conservative Party and the Cultural Politics of Britain, 1929-54, Manchester, Manchester University Press, 2015.

COCKETT Richard, Thinking the Unthinkable : Think-tanks and the Economic CounterRevolution, 1931-1983, Glasgow, Harper Collins, 1995.

DJELIC Marie-Laure, « Spreading Ideas to Change the World: Inventing and Institutionalizing the Neoliberal Think Tank », in GARSTEN Christina et SÖRBOM Adrienne (dir.), Political Affair: Bridging Markets and Politics, Cheltenham, Edward Elgar, 2014, p.1-41.

GARNETT, Mark et DENHAM, Andrew, « A "hollowed-out" tradition? British think tanks in the 21st century », in STONE, Diane et DENHAM, Andrew, Think Tank Traditions : Policy Analysis across Nations, Manchester, Manchester University Press, 2004, p. 232-246.

GARNETT Mark et LORENZONI Virgile, "British think tanks in the time of "Brexit" ", in ABELSON Donald E. et RASTRICK, Christopher (dir.), Handbook on Policy Think Tanks, London, Edward Elgar, 2020 (à paraître).

PIRIE Madsen, Think Tank : The Story of the Adam Smith Institute, Londres, Biteback Publishing Ltd, 2012.

\section{NOTES}

1. BERTHEZÈNE Clarisse, Training Minds for the War of Ideas: Ashridge College, the Conservative Party and the Cultural Politics of Britain, 1929-54, Manchester, Manchester University Press, 2015.

2. COCKETT Richard, Thinking the Unthinkable: Think-tanks and the Economic CounterRevolution, 1931-1983, Glasgow, Harper Collins, 1995.

3. GARNETT, Mark et DENHAM, Andrew, « A "hollowed-out" tradition? British think tanks in the 21st century ", in STONE, Diane et DENHAM, Andrew, Think Tank Traditions : Policy Analysis across Nations, Manchester, Manchester University Press, 2004, p. 232-246.

4. DJELIC Marie-Laure, «Spreading Ideas to Change the World: Inventing and Institutionalizing the Neoliberal Think Tank », in GARSTEN Christina et SÖRBOM Adrienne (dir.), Political Affair: Bridging Markets and Politics, Cheltenham, Edward Elgar, 2014, p.1-41.

5. GARNETT Mark et LORENZONI Virgile, "British think tanks in the time of "Brexit"», in ABELSON Donald E. et RASTRICK, Christopher (dir.), Handbook on Policy Think Tanks, London, Edward Elgar, 2020 (à paraître).

6. Brochure publiée par la Brexit Unit sur le site internet de l'IEA, juin 2017, https://iea.org.uk/ wp-content/uploads/2017/06/Brexit-Unit-introduction-June-2017.pdf

7. David Pegg, Felicity Lawrence et Rob Evans, «Rightwing thinktank breached charity law by campaigning for hard Brexit ", The Guardian, 5 février 2019, https://www.theguardian.com/ politics/2019/feb/05/rightwing-thinktank-breached-charity-law-by-campaigning-for-hardbrexit

8. Jonny Ball, «Announcing a key "Labour Leaver" policy, Johnson steps on the opposition's toes", The New Statesman, 29 novembre 2019, https://www.newstatesman.com/spotlight/ 2019/11/announcing-key-labour-leaver-policy-johnson-steps-opposition-s-toes 
9. Caelainn Barr, Pamela Duncan, Rob Evans, David Pegg et Felicity Lawrence, «How the right's radical thinktanks reshaped the Conservative party », The Guardian, 29 novembre 2019, https:// www.theguardian.com/politics/2019/nov/29/rightwing-thinktank-conservative-boris-johnsonbrexit-atlas-network

10. Kate Andrews, "BBC Question Time from South Fields ", 24 octobre 2019. Cité sur le site internet de l'IEA le 28 octobre 2020, https://iea.org.uk/media/kate-andrews-appears-on-bbcsquestion-time/

11. Kate Andrews et Heidi Chow, « Debate: Is Labour right to warn over US interest in the NHS, uncovered in the leaked trade talk papers?", City A.M., 28 novembre 2019, https:// www.cityam.com/debate-is-labour-right-to-warn-over-us-interest-in-the-nhs-uncovered-in-theleaked-trade-talk-papers/

12. Victoria Hewson, «Jeremy Corbyn's claim that the NHS is 'up for sale' in post-Brexit US trade talks does not stack up », BrexitCentral, 27 novembre 2019, https://brexitcentral.com/jeremycorbyns-claim-that-the-nhs-is-up-for-sale-in-post-brexit-us-trade-talks-does-not-stack-up/

13. Mark Littlewood, "Americans don't want to buy the NHS, but they can help to improve it ", The Times, 2 décembre 2019, https://www.thetimes.co.uk/article/americans-dont-want-to-buythe-nhs-but-they-can-help-to-improve-it-2m0swppfv

14. James Booth, "City reacts with shock to Labour's plan to renationalise BT », City A.M., 14 novembre 2019, https://www.cityam.com/city-reacts-with-shock-to-labours-plans-to-partnationalise-bt/

15. Daniel Martin, Claire Allicott et Matt Oliver, "Crackpot" Corbyn's "communist" plan to nationalise broadband "adds BT to list of companies facing Labour Danger" says expert ", The Daily Mail, 14 novembre 2019, https://www.dailymail.co.uk/news/article-7692355/CrackpotCorbyns-communist-plan-nationalise-broadband.html

16. Site Internet de l'IEA, 16 novembre 2019, https://iea.org.uk/media/whatever-the-purposeborrowing-is-still-borrowing/

17. Kate Andrews, «Labour cash grab would threaten every pensioner ", The Sunday Express, 17 novembre 2019, https://www.express.co.uk/comment/expresscomment/1205498/labourmanifesto-broadband-general-election-2019-free-dentist-Jeremy-corbyn

18. Julian Jessop et Len Shackleton, «Renationalisation : Back to the Future? », site internet de l'IEA, 9 décembre 2019, https://iea.org.uk/publications/renationalisation-back-to-the-future/

19. Edward Malnick, «Labour sought legal advice that Jeremy Corbyn can implement broadband policy if UK remains in EU», The Daily Telegraph, 8 décembre 2019, https:// www.telegraph.co.uk/politics/2019/12/08/labour-sought-legal-advice-jeremy-corbyn-canimplement-broadband/

20. Daniel Martin, «John McDonnell is to unveil Labour's plan to put workers on boards of power firms after they are re-nationalised", The Daily Mail, 8 décembre 2019, https:// www.dailymail.co.uk/news/article-7770357/Labours-plan-workers-boards-power-firmsnationalised.html

21. Kate Devlin, «Labour pledges pay rises in first budget », The Times, 9 décembre 2019, https:// www.thetimes.co.uk/article/labour-pledges-pay-rises-in-first-budget-tbcfpghxs

22. Mark Littlewood, «Tories have a long way to go to prove they are the taxpayers' party ", The Times, 26 novembre 2019, https://www.thetimes.co.uk/edition/comment/tories-have-a-longway-to-go-to-prove-they-are-the-taxpayers-party-q6hh7zmll

23. Manifeste du Parti travailliste 2019, Site Internet du Parti travailliste, https://labour.org.uk/ wp-content/uploads/2019/11/Real-Change-Labour-Manifesto-2019.pdf

24. Manifeste du Parti conservateur 2019, Site internet du Parti conservateur, https://assetsglobal.website-files.com/5da42e2cae7ebd3f8bde353c/ 5dda924905da587992a064ba_Conservative\%202019\%20Manifesto.pdf 
25. Gordon Rayner et Harry Yorke, « Jeremy Corbyn accused of masking true cost of manifesto to ordinary taxpayers ", The Daily Telegraph, 21 novembre 2019, https://www.telegraph.co.uk/ politics/2019/11/21/jeremy-corbyn-accused-masking-true-cost-manifesto-ordinary-taxpayers/ 26. Paul Withers, "Corbyn's "crazy" personal care plan will cost YOU £6BN "Election plot and crippling debt" ", The Daily Express, 8 novembre 2019, https://www.express.co.uk/news/ politics/1202015/general-election-jeremy-corbyn-labour-party-policy-free-personal-caretaxpayer-cost

27. Darren Hunt, « Boris and Corbyn warned pledges could trigger 1970s return - "Serious cause for concern" ", The Daily Express, 4 novembre 2019, https://www.express.co.uk/news/politics/ 1199939/Election-news-Labour-Party-Jeremy-Corbyn-Boris-Johnson-pledges-manifesto-debt

28. Kate Andrews, «The Tories and Labour both seem happy to throw fiscal responsibility to the wind », City A.M., 8 novembre 2019, https://www.cityam.com/the-tories-and-labour-both-seemhappy-to-throw-fiscal-responsibility-to-the-wind/

29. Emily Carver, «It's time for economics liberals to stand up and fight back », 1828, 9 décembre 2019, https://1828uk.com/2019/11/06/its-time-for-economic-liberals-to-stand-up-and-fightback/

30. Voir DJELIC Marie-Laure, «Spreading Ideas to Change the World: Inventing and Institutionalizing the Neoliberal Think Tank », in GARSTEN Christina et SÖRBOM Adrienne (dir.), Political Affair: Bridging Markets and Politics, Cheltenham, Edward Elgar, 2014, p.1-41.

31. Shanker Singham, Barnabas Reynolds, Dan Griswold, Derek Scissors, Ted Bromund, Tom Clougherty, Sheila Lawlor, Matt Kilcoyne, Diana Furchtgott-Roth et Iain Murray, « The Ideal UKUS Agreement: A Free-Trader's Perspective », site internet du Cato Institute, septembre 2018, https://www.cato.org/sites/cato.org/files/pubs/wtpapers/ideal-us-uk-free-trade-agreementupdate.pdf

32. Voir la section "partners» $d u$ site internet de l'Atlas Network, https:// www.atlasnetwork.org/partners

33. Voir le site internet de l'IEA, https://iea.org.uk/events/thinktent-in-conversation-with-rthon-sajid-javid-mp-chancellor-of-the-exchequer/

34. Voir le site internet de l'IEA, https://iea.org.uk/events/thinktent-trade-after-brexit-willfreer-markets-enrich-us/

35. Nick King et Eamonn Ives, «A Rising Tide : Levelling Up Left-behind Britain », site internet du CPS, 29 octobre 2019, https://www.cps.org.uk/files/reports/original/191028170944ARisingTide3.pdf

36. L'idée de la semaine de quatre jours est développée dans un rapport du Progressive Economic Forum (PEF). Voir Lord Skidelsky, « How to achieve shorter working hours », site internet du PEF, 12 septembre 2019, https://progressiveeconomyforum.com/publications/how-to-achieveshorter-working-hours/

37. Jethro Elsden, "The costs of a Four-Day Week to the Public Sector ", site internet du CPS, 5 novembre 2019, https://www.cps.org.uk/research/the-costs-of-a-four-day-week-to-the-publicsector/

38. Rachel Sylvester, Alice Thomson et Henry Zeffman, "General election 2019 : Labour's fourday week "to cost taxpayers £17bn" ", The Times, 5 novembre 2019, https:// www.thetimes.co.uk/article/general-election-2019-labours-four-day-week-to-cost-

taxpayers-17bn-ldrxhzh86

39. Voir le site internet du CPS, https://www.cps.org.uk/

40. Voir le site internet de l'IFT, section « About », http://www.ifreetrade.org/about

41. Sam Knight, " The man who brought you Brexit », The Guardian, 29 septembre 2016, https:// www.theguardian.com/politics/2016/sep/29/daniel-hannan-the-man-who-brought-you-brexit 
42. Daniel Hannan, « Why is "Mr. Brexit" concentrating his tariffs on the UK? ", The Washington Examiner, 28 octobre 2019, https://www.washingtonexaminer.com/opinion/why-is-mr-brexitconcentrating-his-tariffs-on-the-uk

43. Voir le site internet de l'Atlas Network, section "partners in the United States ", https:// www.atlasnetwork.org/partners/global-directory/united-states/5

44. Nile Gardiner, «There will be a UK-US trade deal by 2020 », The Telegraph, 5 novembre 2019, https://www.telegraph.co.uk/politics/2019/11/05/will-uk-us-trade-deal-2020/

45. PIRIE Madsen, Think Tank: The Story of the Adam Smith Institute, Londres, Biteback Publishing Ltd, 2012.

46. Paul Johnson, " Don't expect those carefully costed manifesto promises to become reality », The Times, 9 décembre 2019, https://www.thetimes.co.uk/edition/business/there-is-devil-inthe-detail-but-the-party-manifestos-fail-the-big-picture-too-88k8jgzk8

47. PA Media, "There is such a thing as society, says Johnson from bunker ", The Guardian, 29 mars 2020, https://www.theguardian.com/politics/2020/mar/29/20000-nhs-staff-return-toservice-johnson-says-from-coronavirus-isolation

\section{RÉSUMÉS}

Cet article se propose d'analyser le rôle joué par plusieurs think tanks britanniques (notamment l'Institute of Economic Affairs, le Centre for Policy Studies et l'Initiative for Free Trade) dans l'élection législative de décembre 2019 au Royaume-Uni, une élection dominée par le sujet du Brexit. Ces dernières décennies le modèle originel du think tank britannique, reposant sur des propositions de politiques publiques émanant d'un travail de recherche indépendant, se voit concurrencé par l'émergence d'organisations fortement colorées idéologiquement, qui prétendent au statut de think tank mais semblent plus intéressées par la visibilité médiatique que par le développement d'une pensée rigoureuse. Nous qualifions ici ce modèle de think tank évidé. Nous nous penchons ici sur le cas de ceux qui se situent à la droite du spectre politique et se consacrent à la promotion du libre-échange. Pour cela, ils s'appuient sur un système agressif de promotion, une maîtrise des tropes et des codes médiatiques, ainsi que sur la construction d'un discours simplifié, séduisant et parfois trompeur, bâti autour d'une promesse d'innovation. Nous examinons ici la participation de ces think tanks au débat électoral de 2019, et notamment leur activité dans les médias. Nous entendons démontrer que les think tanks évidés ne sont peutêtre pas aussi adaptés à l'ère du Brexit (et à la politique populiste) que l'on pourrait le supposer. En effet, les think tanks idéologiques ont-ils toujours un rôle utile à jouer, au sein d'un discours de plus en plus polarisé entre la présentation des faits et la diffusion d'informations fortement orientées?

This article analyses the role played by several British think tanks (including the Institute of Economic Affairs, the Centre for Policy Studies, and the Initiative for Free Trade) in the run-up to the December 2019 general election, which was dominated by the issue of Brexit. In recent decades, the original British think tank model, based on the idea of policy advice arising from independent research, has been challenged by the emergence of strongly ideological bodies, who claim the label of think tanks but seem more interested in attracting positive media coverage than in rigorous thinking. This different think tank model is here referred to as hollowed out. We focus on right-wing organisations, primarily concerned with the promotion of free trade, which 
rely on aggressive advocacy, mastery of media tropes and codes, and the construction of a simplified and sometimes beguiling discourse, built on promises of innovation. We shall examine the attempts by those think tanks to make their voices heard during the 2019 election. We seek to demonstrate that the hollowed-out think tanks might not be as suited to the era of Brexit and populist politics as one might suppose. Indeed, the article will ask whether the ideological think tanks still have a useful role to play, in a discourse which is increasingly polarised between the purveyors of facts and false news.

\section{INDEX}

Mots-clés : think tanks, élections législatives, Brexit, médias, libéralisme économique, libreéchange, conservatisme

Keywords : think tanks, general election, Brexit, media, economic liberalism, free trade, conservatism

\section{AUTEURS}

\section{MARK GARNETT}

Department of Politics, Philosophy and Religion, Université de Lancaster, LA1 4YL Lancaster

\section{VIRGILE LORENZONI}

Laboratoire d'Études et de Recherche sur le Monde Anglophone (UR 853), Aix-Marseille

Université, 13100 Aix-en-Provence 